\title{
Euler and Infinite Series
}

\section{Morris Kline}

Mathematics Magazine, Vol. 56, No. 5. (Nov., 1983), pp. 307-314.

Stable URL:

http://links.jstor.org/sici?sici=0025-570X\%28198311\%2956\%3A5\%3C307\%3AEAIS\%3E2.0.CO\%3B2-M

Mathematics Magazine is currently published by Mathematical Association of America.

Your use of the JSTOR archive indicates your acceptance of JSTOR's Terms and Conditions of Use, available at http://www.jstor.org/about/terms.html. JSTOR's Terms and Conditions of Use provides, in part, that unless you have obtained prior permission, you may not download an entire issue of a journal or multiple copies of articles, and you may use content in the JSTOR archive only for your personal, non-commercial use.

Please contact the publisher regarding any further use of this work. Publisher contact information may be obtained at http://www.jstor.org/journals/maa.html.

Each copy of any part of a JSTOR transmission must contain the same copyright notice that appears on the screen or printed page of such transmission.

The JSTOR Archive is a trusted digital repository providing for long-term preservation and access to leading academic journals and scholarly literature from around the world. The Archive is supported by libraries, scholarly societies, publishers, and foundations. It is an initiative of JSTOR, a not-for-profit organization with a mission to help the scholarly community take advantage of advances in technology. For more information regarding JSTOR, please contact support@jstor.org. 


\section{Euler and Infinite Series}

\section{Morris KuINe}

Courant Institute of Mathematical Sciences

New York, NY 10012

The history of mathematics is valuable as an account of the gradual development of the many current branches of mathematics. It is extremely fascinating and instructive to study even the false steps made by the greatest minds and in this way reveal their often unsuccessful attempts to formulate correct concepts and proofs, even though they were on the threshold of success. Their efforts to justify their work, which we can now appraise with the advantage of hindsight, often border on the incredible.

These features of history are most conspicuous in the work of Leonhard Euler, the key figure in 18th-century mathematics, and one who should be ranked with Archimedes, Newton, and Gauss. Euler's recorded work on infinite series provides a prime example of the struggles, successes and failures which are an essential part of the creative life of almost all great mathematicians. The few examples discussed in this paper will serve to illustrate how Euler surmounted the difficulties he encountered.

Euler first undertook work on infinite series around 1730, and by that time, John Wallis, Isaac Newton, Gottfried Leibniz, Brook Taylor, and Colin Maclaurin had demonstrated the series calculation of the constants $e$ and $\pi$ and the use of infinite series to represent functions in order to integrate those that could not be treated in closed form. Hence it is understandable that Euler should have tackled the subject. Like his predecessors, Euler's work lacks rigor, is often ad hoc, and contains blunders, but despite this, his calculations reveal an uncanny ability to judge when his methods might lead to correct results. Our discussion will not follow the precise historical order of Euler's investigations of series; he made contributions throughout his lifetime.

To appreciate the first example of Euler's work on series, we must consider some background. A series which caused endless dispute was

$$
1-1+1-1+\cdots .
$$

It seemed clear that by writing this series as

$$
(1-1)+(1-1)+(1-1)+\cdots
$$

the sum should be 0 . It seemed equally clear, however, that by writing the series as

$$
1-(1-1)-(1-1)-\cdots
$$

the sum should be 1 . But still another sum seemed as reasonable. If $S$ denotes the sum of the series (1), then

$$
S=1-(1-1+1-1+\cdots)=1-S .
$$

Hence $S=\frac{1}{2}$. This value was also supported by the formula for summing a geometric series with common ratio -1 .

Guido Grandi (1671-1742), in his little book Quadratura circula et hyperbolae per infinitas hyperbolas geometrice exhibita (1703), obtained the third result by a variant of the geometric series argument, using the binomial expansion

$$
\frac{1}{1+x}=1-x+x^{2}-x^{3}+\cdots,
$$

with $x=1$. (He also argued that since the sum was both 0 and $\frac{1}{2}$, he had proved that the world could be created out of nothing.) In a letter to Christian Wolf published in the Acta eruditorum of 1713, Leibniz agreed with Grandi's result but thought that it should be possible to obtain it without resorting to the function $1 /(1+x)$. He argued that, since the successive partial sums are 
$1,0,1,0,1, \cdots$, with 1 and 0 equally probable, one should therefore take $\frac{1}{2}$, the arithmetic mean, as the sum. This argument was accepted by James, John and Daniel Bernoulli. Leibniz conceded that his argument was more metaphysical than mathematical, but said that there is more metaphysical truth in mathematics than is generally recognized.

Euler took a hand in this argument. To obtain the sum of the series (1), he argued in a manner similar to Grandi, substituting $x=-1$ in the expansion

$$
1 /(1-x)=1+x+x^{2}+\cdots
$$

and obtained

$$
\frac{1}{2}=1-1+1-1+\cdots .
$$

At this early stage of his work on series, Euler used expansion of functions into series to sum other divergent series. For example, he substituted $x=-1$ in the expansion

$$
1 /(1+x)^{2}=(1+x)^{-2}=1-2 x+3 x^{2}-4 x^{3}+\cdots
$$

and obtained

$$
\infty=1+2+3+4+\cdots .
$$

To Euler, this seemed reasonable; he treated infinity as a number. He then considered the geometric (or binomial) series for $1 /(1-x)$ with $x=2$ and obtained

$$
-1=1+2+4+8+\cdots \text {. }
$$

Since the terms of series (3) exceed the corresponding terms of series (2), Euler concluded that the sum -1 is larger than infinity. Some of Euler's contemporaries argued that negative numbers larger than infinity are different from those less than 0 . Euler objected and argued that infinity separates positive and negative numbers just as 0 does.

In a paper of 1734/35 [7], Euler started with the series

$$
y=\sin x=x-x^{3} / 3 !+x^{5} / 5 !+\cdots
$$

and rewrote the equation in the form

$$
1-x / y+x^{3} / 3 ! y+x^{5} / 5 ! y-\cdots=0 .
$$

He then treated the left side of (5) as an infinite polynomial and argued as follows. (The argument is based on the fact that the sum of the reciprocals of the roots of the polynomial $p(x)=1-a_{1} x$ $+a_{2} x^{2}-a_{3} x^{3}+\cdots+(-1)^{k} a_{k} x^{k}$ is $a_{1}$, the sum of the squares of the reciprocals of the roots of $p(x)$ is $a_{1}{ }^{2}-2 a_{2}$, and so on, for higher roots.) Let $A, B, C, \cdots$ be solutions of equation (5). Then the polynomial can be factored into an infinite product,

$$
1-\frac{x}{y}+\frac{x^{3}}{3 ! y}-\frac{x^{5}}{5 ! y}+\cdots=\left(1-\frac{x}{A}\right)\left(1-\frac{x}{B}\right)\left(1-\frac{x}{C}\right) \cdots
$$

If $A$ is the smallest value of $x$ whose sine is $y$, then all other solutions $B, C, \cdots$ are $\pi-A, 2 \pi+$ $A, 3 \pi-A, \cdots ;-\pi-A,-2 \pi+A,-3 \pi-A, \cdots$. Thus

$$
\begin{array}{r}
\frac{1}{A}+\frac{1}{\pi-A}+\frac{1}{2 \pi+A}+\cdots-\frac{1}{\pi+A}-\frac{1}{2 \pi-A}-\frac{1}{3 \pi+A}-\cdots=\frac{1}{y} . \\
\frac{1}{A^{2}}+\frac{1}{(\pi-A)^{2}}+\frac{1}{(2 \pi+A)^{2}}+\cdots+\frac{1}{(\pi+A)^{2}}+\frac{1}{(2 \pi+A)^{2}}+\frac{1}{(3 \pi+A)^{2}}+\cdots=\frac{1}{y^{2}}
\end{array}
$$

and so on for higher powers of the reciprocals. If, in equations (4) and (5), we take $y=1$, then $A=\pi / 2$, so that (6) becomes

$$
\frac{4}{\pi}\left(1-\frac{1}{3}+\frac{1}{5}-\frac{1}{7}+\cdots\right)=1
$$

or 


$$
1-\frac{1}{3}+\frac{1}{5}-\frac{1}{7}+\cdots=\frac{\pi}{4}
$$

and (7) becomes

$$
\frac{8}{\pi^{2}}\left(1+\frac{1}{9}+\frac{1}{25}+\frac{1}{49}+\cdots\right)=1
$$

or

$$
1+\frac{1}{3^{2}}+\frac{1}{5^{2}}+\frac{1}{7^{2}}+\cdots=\frac{\pi^{2}}{8} .
$$

Other series that were "summed" in the same manner are

$$
\begin{aligned}
& 1 / 1^{3}-1 / 3^{3}+1 / 5^{3}-\cdots=\frac{\pi^{3}}{32}, \\
& 1 / 1^{4}+1 / 3^{4}+1 / 5^{4}+\cdots=\frac{\pi^{4}}{96}, \\
& 1 / 1^{5}-1 / 3^{5}+1 / 5^{5}-\cdots=\frac{5 \pi^{5}}{1536}, \\
& 1 / 1^{6}+1 / 3^{6}+1 / 5^{6}+\cdots=\frac{\pi^{6}}{960},
\end{aligned}
$$

and so on. From these series he deduced others. For example, since

$$
\left(1+\frac{1}{2^{2}}+\frac{1}{3^{2}}+\frac{1}{4^{2}}+\cdots\right)-\left(1+\frac{1}{3^{2}}+\frac{1}{5^{2}}+\frac{1}{7^{2}}+\cdots\right)=\frac{1}{2^{2}}\left(1+\frac{1}{2^{2}}+\frac{1}{3^{2}}+\frac{1}{4^{2}}+\cdots\right),
$$

one can use (9) to obtain

$$
1 / 1^{2}+1 / 2^{2}+1 / 3^{2}+1 / 4^{2}+\cdots=\frac{\pi^{2} / 8}{3 / 4}=\frac{\pi^{2}}{6},
$$

and in a similar manner, obtain

$$
1 / 1^{4}+1 / 2^{4}+1 / 3^{4}+1 / 4^{4}+\cdots=\frac{\pi^{4}}{90}
$$

and other sums. R. Ayoub [1] discusses Euler's use of (4) to compute such sums, W. F. Eberlein [3] discusses Euler's use of the infinite product for the sine function, and H. H. Goldstine [9, 3.1, 3.2] indicates Euler's expansions of such functions as $\frac{1}{2}\left(e^{x}-e^{-x}\right)$ and the use of these expansions in computing sums such as (9).

Euler's attempts to sum the reciprocals of powers of the positive integers were not completely idle. In another paper of the same period [4], Euler made a somewhat bizarre use of infinitesimal calculus to find the difference between the sum of the harmonic series and the logarithm, a difference whose expansion utilizes precisely these series of powers. Let

$$
s=1+\frac{1}{2}+\frac{1}{3}+\cdots+\frac{1}{n-1} .
$$

If we regard $n$ as infinite, then 1 is an infinitesimal and we can write $d s=1 / n=1 / n d n$. An integration yields

$$
s=\log n+C .
$$

To find $C$, note that

$$
\frac{1}{x}=\log \left(1+\frac{1}{x}\right)+\frac{1}{2 x^{2}}-\frac{1}{3 x^{3}}+\frac{1}{4 x^{4}}-\frac{1}{5 x^{5}}+\cdots .
$$

Setting $x=1,2,3, \ldots, n-1$, in turn, and adding the $n-1$ equations yields 


$$
\begin{aligned}
1+\frac{1}{2}+\frac{1}{3}+\cdots+\frac{1}{n-1}= & \log n+\frac{1}{2}\left(1+\frac{1}{4}+\frac{1}{9}+\cdots+\frac{1}{(n-1)^{2}}\right) \\
& -\frac{1}{3}\left(1+\frac{1}{8}+\frac{1}{27}+\cdots+\frac{1}{(n-1)^{3}}\right) \\
& +\frac{1}{4}\left(1+\frac{1}{16}+\frac{1}{81}+\cdots+\frac{1}{(n-1)^{4}}\right) \\
& -\cdots
\end{aligned}
$$

The limiting value $\gamma$ of $C$ as $n$ becomes infinite is today called Euler's constant.

In a paper of 1740 [6], Euler obtained one of his finest triumphs, namely,

$$
s_{2 n}=\sum_{\nu=1}^{\infty} \frac{1}{\nu^{2 n}}=(-1)^{n-1} \frac{(2 \pi)^{2 n}}{2(2 n) !} B_{2 n},
$$

where the $B_{2 n}$ are the Bernoulli numbers (see below). The connection with the Bernoulli numbers was actually established a little later in his Institutiones calculi differentialis of 1755 [8]. In the 1740 paper he also determined the sum $\sum_{\nu=1}^{\infty}(-1)^{\nu-1}\left(1 / \nu^{n}\right)$ for the first few odd values of $n$.

In Ars conjectandi (1713), James Bernoulli, who was treating the subject of probability, had introduced the now widely used Bernoulli numbers. Bernoulli had given the following formula for the sum of powers of consecutive positive integers without demonstration:

$$
\begin{aligned}
\sum_{k=1}^{n} k^{c}= & \frac{1}{c+1} n^{c+1}+\frac{1}{2} n^{c}+\frac{c}{2} B_{2} n^{c-1}+\frac{c(c-1)(c-2)}{2 \cdot 3 \cdot 4} B_{4} n^{c-3} \\
& +\frac{c(c-1)(c-2)(c-3)(c-4)}{2 \cdot 3 \cdot 4 \cdot 5 \cdot 6} B_{6} n^{c-5}+\cdots .
\end{aligned}
$$

This series terminates at the last positive power of $n$, and the $B$ 's are the Bernoulli numbers:

$$
B_{2}=1 / 6, B_{4}=-1 / 30, B_{6}=1 / 42, B_{8}=-1 / 30, B_{10}=5 / 66, \ldots .
$$

Bernoulli also gave the recurrence relation which permits one to calculate these coefficients.

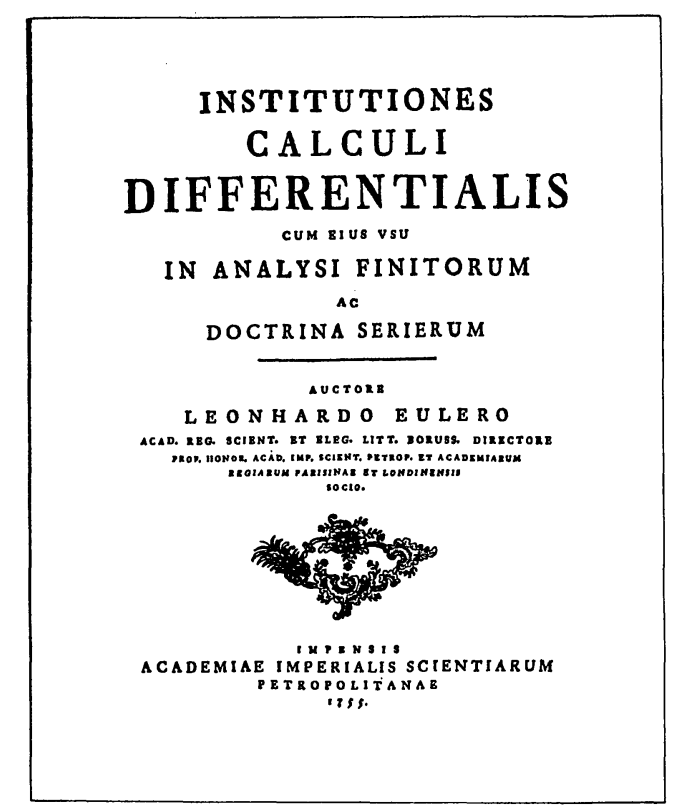


Another famous result of Euler's, the Euler-Maclaurin summation formula, is a generalization of Bernoulli's formula (10). Let $f(x)$ be a real-valued function of the real variable $x$ with $2 k+1$ continuous derivatives on the interval $[0, n]$. Then (in modern notation) Euler's formula is

$$
\begin{aligned}
\sum_{i=0}^{n} f(i)= & \int_{0}^{n} f(x) d x+\frac{1}{2}[f(n)+f(0)]+\frac{B_{2}}{2 !}\left[f^{\prime}(n)-f^{\prime}(0)\right] \\
& +\frac{B_{4}}{4 !}\left[f^{\prime \prime \prime}(n)-f^{\prime \prime \prime}(0)\right]+\cdots \\
& +\frac{B_{2 k}}{(2 k) !}\left[f^{(2 k-1)}(n)-f^{(2 k-1)}(0)\right]+R_{k},
\end{aligned}
$$

where

$$
R_{k}=\int_{0}^{n} f^{(2 k+1)}(x) P_{2 k+1}(x) d x .
$$

Here $n$ and $k$ are positive integers, and $P_{2 k+1}(x)$ is the $(2 k+1)$ th Bernoulli polynomial (which also appears in Bernoulli's Ars conjectandi). It can be represented for $0 \leqslant x \leqslant 1$ by

$$
P_{2 k+1}(x)=2(-1)^{k+1} \sum_{m=1}^{\infty} \frac{\sin (2 \pi m x)}{(2 m \pi)^{2 k+1}} .
$$

The Bernoulli numbers $B_{i}$ are related to the Bernoulli polynomials by

$$
P_{k}(x)=\frac{x^{k}}{k !}+\frac{B_{1} x^{k-1}}{1 !(k-1) !}+\frac{B_{2} x^{k-2}}{2 !(k-2) !}+\cdots+\frac{B_{k}}{k !},
$$

where $B_{1}=-\frac{1}{2}$, and $B_{2 k+1}=0$ for $k=1,2, \cdots$. They are often defined today by a relation given later by Euler, namely,

$$
t\left(e^{t}-1\right)^{-1}=\sum_{i=0}^{\infty} B_{i} \frac{t^{i}}{i !}
$$

Euler's derivation of formula (11) is interesting in its use of the infinitesimal calculus in treating finite series. He begins by noting that if $s(n)=\sum_{i=0}^{n} f(i)$, then

$$
f(n)=s(n)-s(n-1)=-[s(n-1)-s(n)]=\frac{d s}{d n}-\frac{1}{2 !} \frac{d^{2} s}{d n^{2}}+\frac{1}{3 !} \frac{d^{3} s}{d n^{3}}-\cdots ;
$$

hence (solving for $d s / d n$ and integrating),

$$
s=\int f d n+\frac{1}{2 !} \frac{d s}{d n}-\frac{1}{3 !} \frac{d^{2} s}{d n^{2}}+\cdots .
$$

In order to express the sum $s$ in terms of $f$, recursion is used. Differentiating (12) repeatedly gives

$$
\begin{array}{cc}
\frac{d f}{d n}=\frac{d^{2} s}{d n^{2}}-\frac{1}{2 !} \frac{d^{3} s}{d n^{3}}+\frac{1}{3 !} \frac{d^{4} s}{d n^{4}}-\cdots, & \text { so } \frac{d^{2} s}{d n^{2}}=\frac{d f}{d n}+\frac{1}{2 !} \frac{d^{3} s}{d n^{3}}-\cdots \\
\frac{d^{2} f}{d n^{2}}=\frac{d^{3} s}{d n^{3}}-\frac{1}{2 !} \frac{d^{4} s}{d n^{4}}+\frac{1}{3 !} \frac{d^{5} s}{d n^{5}}-\cdots, & \text { so } \frac{d^{3} s}{d n^{3}}=\frac{d^{2} f}{d n^{2}}+\frac{1}{2 !} \frac{d^{4} s}{d n^{4}}-\cdots
\end{array}
$$

and so on. Substituting these values for $d s / d n, d s^{2} / d n^{2}, d s^{3} / d n^{3}, \cdots$ in (12) gives

$$
\begin{aligned}
s= & \int f d n+\frac{1}{2 !}\left[f+\frac{1}{2 !}\left(\frac{d f}{d n}+\frac{1}{2 !}\left(\frac{d f^{2}}{d n^{2}}+\cdots\right)\right)-\frac{1}{3 !}\left(\frac{d^{2} f}{d n^{2}} \cdots\right)\right] \\
& -\frac{1}{3 !}\left(\frac{d f}{d n}+\frac{1}{2 !}\left(\frac{d^{2} f}{d n^{2}}+\cdots\right)+\cdots\right)+\frac{1}{4 !}\left(\frac{d^{2} f}{d n^{2}}-\cdots\right)-\cdots
\end{aligned}
$$


which is cumbersome, but does show the form in which $s$ can be expressed. Euler wrote

$$
s=\int f d n+\alpha f+\frac{\beta d f}{d n}+\frac{\gamma d^{2} f}{d n^{2}}+\frac{\delta d^{3} f}{d n^{3}}+\cdots
$$

and substituted $s$ and its derivatives into (13) to obtain recursion relations for the coefficients of $f, d f / d n, d^{2} f / d n^{2}$, etc., finally obtaining formula (11). A discussion of Euler's derivation of the Euler-Maclaurin formula as well as some of his interesting applications of it is contained in [9,3.3,3.4]; a modern summary of Euler's work on the formula is contained in [1, p. 1074].

If $n$ is allowed to go to infinity in the Euler-Maclaurin formula (11), the infinite series is divergent for almost all $f(x)$ which occur in applications. Nevertheless, under modest additional hypotheses, the remainder $R_{k}$ is less than the first term neglected, and so the series and the integral give useful approximations to each other, depending on which is easier to compute.

Independently of Euler, Maclaurin (Treatise on Fluxions, 1742) arrived at the same summation formula but by a method a little surer and closer to that which we use today. The remainder was first added and seriously treated by Poisson.

Euler also introduced in his Institutiones of 1755 a transformation of series, still known and used [12]. Given a series $\sum_{n=0}^{\infty} b_{n}$, he wrote it as $\sum_{n=0}^{\infty}(-1)^{n} a_{n}$. Then by a number of formal algebraic steps he showed that

$$
\sum_{n=0}^{\infty}(-1)^{n} a_{n}=\sum_{n=0}^{\infty}(-1)^{n} \frac{\Delta^{n} a_{0}}{2^{n+1}}
$$

where

$$
\Delta^{0} a_{0}=a_{0}, \Delta^{1} a_{0}=a_{1}-a_{0}, \Delta^{n} a_{0}=\Delta^{n-1} a_{1}-\Delta^{n-1} a_{0}=\sum_{i=0}^{n}(-1)^{n-i}\left(\begin{array}{c}
n \\
i
\end{array}\right) a_{i}, n \geqslant 2 .
$$

His derivation of (15) is as follows. Let $a_{n}=(-1)^{n} b_{n}$ and introduce variables $x$ and $y$ related by $x=y /(1-y)=y+y^{2}+y^{3}+\cdots$. Then

$$
\begin{aligned}
b_{0} x & +b_{1} x^{2}+b_{2} x^{3}+\cdots \\
= & a_{0} x-a_{1} x^{2}+a_{2} x^{3}-a_{3} x^{4}+\cdots \\
= & a_{0}\left(y+y^{2}+y^{3}+\cdots\right)-a_{1}\left(y^{2}+2 y^{3}+3 y^{4}+4 y^{5}+\cdots\right) \\
& +a_{2}\left(y^{3}+3 y^{4}+6 y^{5}+10 y^{6}+\cdots\right)-a_{3}\left(y^{4}+4 y^{5}+10 y^{6}+20 y^{7}+\cdots\right)+\cdots \\
= & a_{0} y-\left(a_{1}-a_{0}\right) y^{2}+\left(a_{2}-2 a_{1}+a_{0}\right) y^{3}-\cdots .
\end{aligned}
$$

Setting $x=1$ and $y=\frac{1}{2}$ yields

$$
\sum_{n=0}^{\infty} b_{n}=a_{0}-a_{1}+a_{2}-a_{3}+\cdots=\frac{a_{0}}{2}-\frac{\Delta a_{0}}{4}+\frac{\Delta^{2} a_{0}}{8}-\cdots,
$$

as required.

The transformation in (15) often converts a convergent series into a more rapidly converging one. However, for Euler, who did not usually distinguish between convergent and divergent series, the transformation could also transform divergent series into convergent ones. For, if one applies (15) to the series (1), which is $\sum_{n=0}^{\infty}(-1)^{n}$, then since $a_{0}=1$ and $\Delta^{n} a_{0}=0$ for all $n>1$, the sum on the right is $1 / 2$. Likewise for the series

$$
1-2+2^{2}-2^{3}+2^{4} \cdots
$$

the transformation in (15) gives

$$
\sum_{n=0}^{\infty}(-1)^{n} 2^{n}=\frac{1}{2}(1)+\frac{1}{4}(-1)+\frac{1}{8}(1)-\frac{1}{16}(-1)+\cdots=\frac{1}{3} .
$$




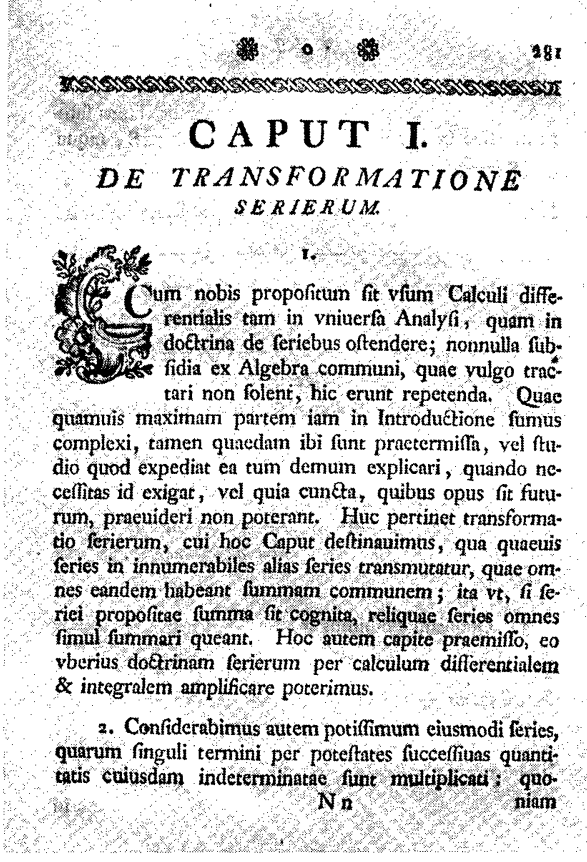

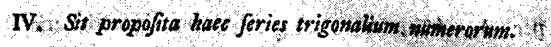
$S=1-3+6-10+15-21+2 d$

Diff. $\mathrm{x}=2,3,4,3,6,8 \mathrm{c}$

Diff. $2=1, \quad x, \quad x, \quad 1, \quad \& c$.

Hic ergo ob $a=1, \Delta a=2, \& \Delta \Delta a=1 ;$ crit $s=1-1+1=r$

V. sit propofite feries quadratorwn:

$\mathrm{S}=1-4+9-16+25-36+8 \mathrm{c}$

Diff. $x=3,5,2,2,3,1, \quad \& c$

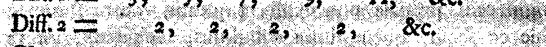

$\mathrm{Ob} a=1 ; \Delta a=3, \triangle \Delta_{a}=2 ;$ erit $\mathrm{S}=1-1+\mathrm{a}=0$

VI. Sit propofita feries biquadraterum

$S=x-16+81-256+625-1296+8 c$

Diff $x=15, \quad 65, \quad 175, \quad 369, \quad 671$

Diff $2=\quad 50,110,194,302,60$

Diff. $3=60,84,108$

Diff $4=24,24$. 24,

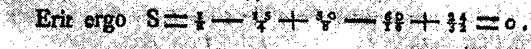

ro. Si feries magis diuergant vit geometriae aliaeque fimiles, eae hoc modo ftatim in feriem magis convergentem transmutantur, quae nifi adhuc fatis conuergat, eodem modo in aliam magis conuergentem con vertern.

(1)

L S

Euler demonstrates (Institutiones, Pars Posterior, Chap. I) his transformation of series with many examples. Here he "sums" the alternating series of triangular numbers, $\sum_{n=0}^{\infty}(-1)^{n}(n+1)(n+2) / 2=1 / 8$, the alternating series of squares, $\sum_{n=0}^{\infty}(-1)^{n}(n+1)^{2}=0$ and of fourth powers $\sum_{n=0}^{\infty}(-1)^{n}(n+1)^{4}=0$.

These results are the same as those Euler got by taking the sum of the series to be the value of the function from which the series is derived.

Euler took up the subject of sums of series in a major paper of 1754/55 entitled "On Divergent Series" [5], in which he recognized the distinction between convergent and divergent series. Apropos of the former he says that for those series in which by constantly adding terms we approach closer and closer to a fixed number, which happens when the terms continually decrease, the series is said to be convergent and the fixed number is its sum. Series whose terms do not decrease and may even increase are divergent.

On divergent series, Euler says one should not use the term "sum" because this refers to actual addition. Euler then states a general principle which explains what he means by the definite value of a divergent series. He points out that the divergent series comes from finite algebraic expressions and then says that the value of the series is the value of the algebraic expression from which the series comes. Euler further states, "Whenever an infinite series is obtained as the development of some closed expression, it may be used in mathematical operations as the equivalent of that expression, even for values of the variable for which the series diverges." $\mathrm{He}$ repeats this principle in his Institutiones of 1755:

Let us say, therefore, that the sum of any infinite series is the finite expression, by the expansion of which the series is generated. In this sense the sum of the infinite series $1-x+x^{2}-x^{3}+\cdots$ will be $1 /(1+x)$, because the series arises from the expansion of the fraction, whatever number is put in place of $x$. If this is agreed, the new definition of the word sum coincides with the ordinary meaning when a series converges; and since divergent series have no sum in the proper sense of the word, no inconvenience can arise from this terminology. Finally, by means of this definition, we can preserve the utility of divergent series and defend their use from all objections.

It is fairly certain that Euler meant to limit this doctrine to power series. 
Other 18th-century mathematicians also recognized that a distinction must be made between what we now call convergent and divergent series, though they were not at all clear as to what the distinction should be. They were dealing with a new concept and, like all pioneers, they had to struggle to clear the forest. Certainly the interpretation of series suggested by Newton, and adopted by Leibniz, Euler, and Lagrange, that series are just long polynomials and so belong in the domain of algebra, could not serve as a rigorous foundation for the work with series.

One outstanding characteristic of the 18th-century investigations is that mathematicians trusted the symbols far more than logic. Because infinite series had the same symbolic form for all values of $x$, the distinction between values of $x$ for which the series converged and values for which they diverged did not seem to demand much attention. And even though it was recognized that some series, such as $1+2+3+\cdots$, had infinite sums, mathematicians preferred to try to give meaning to the sums rather than question the applicability of summation. Of course, they were fully aware of the need for some proofs. We have seen that Euler did try to justify his use of divergent series. But the few efforts to achieve rigor, significant because they show that standards of rigor vary with the times [10], did not validate the work of the century, and mathematicians almost willingly took the position that what cannot be cured must be endured.

Though we have only glimpsed some of Euler's work, almost all of the great mathematicians of the 18th century contributed to the subject of infinite series [13]. It is fair to say that in this work the formal view dominated. Aware of the power of formal manipulation, mathematicians either ignored or deferred consideration of any limitations to their techniques, such as the importance of convergence. Their work produced useful results, and they were satisfied with this pragmatic sanction. They exceeded the bounds of what they could justify, but they were at least prudent in their use of divergent series. However, these 18th-century mathematicians were to have the last word. Dimly, they saw in divergent infinite series, ideas which were later to gain acceptance, namely, summability and asymptotic series [2], [11], [13, chapter 47], [14].

I wish to thank Professor Edward J. Barbeau of the University of Toronto for his critique and for supplying some material on Euler's proofs.

\section{References}

[1] Raymond Ayoub, Euler and the zeta function, Amer. Math. Monthly, 81 (1974) 1067-1087.

[2] E. J. Barbeau, Euler subdues a very obstreperous series, Amer. Math. Monthly, 86 (1979) 356-372.

[ 3 ] W. F. Eberlein, On Euler's infinite product for the sine, J. Math. Anal. Appl., 58 (1977) 147-151.

[4] L. Euler, De progressionibus harmonicis observationes, Comm. acad. sci. Petrop., 7 (1734/35), p. 150-161 = Opera Omnia, (1) 14, 87-100.

[ 5 ] - De seriebus divergentibus, Novi comm. acad. sci. Petrop., $5(1754 / 55), 1760$, pp. 205-237= Opera Omnia, (1) 14, 585-617. An English translation by E. J. Barbeau and P. J. Leah is in Historia Math., 3 (1976) $141-160$.

[ 6 ] - De seriebus quibusdam considerationes, Comm. acad. sci. Petrop., 12 (1740), 1750, pp. 53-96 = Opera Omnia, (1) 14, 407-462.

[ 7 ] , De summis serierum reciprocarum, Comm. acad. sci. Petrop., $7(1734 / 35), 1740$, pp. 123-134 = Opera Omnia, (1) 14, 73-86.

[ 8 ] Institutiones calculi differentialis cum lius usu in analysi finitorum ac doctrina serierum, Acad. Imp. Sci., Petrop. = Opera Omnia, (1) 10, 309-336.

[ 9 ] H. H. Goldstine, A History of Numerical Analysis from the 16th through the 19th Century, Springer-Verlag, 1977, especially Chapter 3.

[10] J. V. Grabiner, Is mathematical truth time-dependent?, Amer. Math. Monthly, 81 (1974) 354-365.

[11] G. H. Hardy, Divergent Series, Oxford University Press, 1949.

[12] R. Johnsonbaugh, Summing an alternating series, Amer. Math. Monthly, 86 (1979) 637-648.

[13] Morris Kline, Mathematical Thought from Ancient to Modern Times, Oxford University Press, 1972, especially Chapter 20 .

[14] John Tucciarone, The development of the theory of summable divergent series, Arch. Hist. Exact Sci., 10 (1973) $1-40$. 